\title{
Galectin-9 Expression Profile in Patients with Gastric Cancer and Peptic Ulcer Disease
}

\author{
Mahdieh Naghavi-Alhosseini ${ }^{1,2}$, Tarang Taghvaei ${ }^{3}$, Hossein Asgarian-Omran ${ }^{1,4 *}$ \\ 1 Department of Immunology, School of Medicine, Mazandaran University of Medical Sciences, Sari, Iran. \\ 2 Molecular and Cell Biology Research Center, School of Medicine, Mazandaran University of Medical Sciences, Sari, Iran. \\ 3 Department of Internal Medicine, Imam Khomeini Hospital, Mazandaran University of Medical Sciences, Sari, Iran. \\ 4 Immunogenetic Research Center, School of Medicine, Mazandaran University of Medical Sciences, Sari, Iran.
}

\section{Received: 5 Sep 2016}

Revised : 28 Sep 2016

Accepted: 15 Oct 2016

Corresponding Author:

Hossein Asgarian-Omran

Department of Immunology, School of Medicine, Mazandaran University of

Medical Sciences, Sari, Iran

Phone: +98 1133543081

Fax: +98 1133543249

E-mail: asgarianhossein@yahoo.com

\begin{abstract}
Background: Chronic inflammation and dysregulation of the immune system mechanisms are now well established as primary triggers to gastric cancer and peptic ulcer disease. Galectin-9 (Gal-9) is a member of the galectin family and known as an inducer of cell aggregation, adhesion and apoptosis. Gal-9 interaction with its main ligand T-cell immunoglobulin and mucin domain protein-3 (Tim-3) leads to the apoptosis of $\mathrm{T}$ cells and dys-regulation of the immune responses in different tumors. In the current study, the mRNA expression pattern of Gal-9 was evaluated in gastric biopsies of patients with gastric cancer and peptic ulcer disease.

Materials and Methods: In this case control study, gastric biopsies were obtained from 46 patients with gastric cancer, 44 patients with peptic ulcer disease and 41 cases with non-ulcer dyspepsia served as controls who underwent endoscopy for the evaluation of their gastric problems. Infection with Helicobacter pylori was determined by rapid urease test for all participants and $\mathrm{H} \& \mathrm{E}$ staining for GC patients. Total RNA was extracted from all gastric tissues and used for cDNA synthesis. Relative expression of Gal-9 mRNA was determined by Real-time PCR using $\beta$-actin as a housekeeping gene.

Results: Gal-9 was similarly expressed in all three studied groups. No statistical difference was found for Gal-9 expression between gastric cancer patients and control group ( $2-\Delta \mathrm{Ct}=0.022$ vs $0.0144, \mathrm{p}=0.30)$ and also between peptic ulcer and control groups $(2-\Delta \mathrm{Ct}=0.088$ vs $0.144, \mathrm{p}=0.16)$. No correlation was found between Gal-9 expression and infection with Helicobacter pylori $(2-\Delta \mathrm{Ct}=0.1 \mathrm{vs}$ $0.129, \mathrm{p}=0.51$ ).

Conclusion: Similar expression of Gal-9 in gastric tissues from patients with gastric cancer, peptic ulcer and also non-ulcer dyspepsia individuals suggests no possible role of this molecule on tumorigenesis and immunoregulatory mechanisms of these gastric disorders.
\end{abstract}

Keywords: Gastric cancer; Peptic ulcer disease; Galectin-9; Helicobacter pylori

Please cite this article as: Naghavi-Alhosseini M, Taghvaei T, Asgarian-Omran H. Galectin-9 Expression Profile in Patients with Gastric Cancer and Peptic Ulcer Disease. Res Mol Med. 2016; 4 (4): 1-7

\section{Introduction}

Gastric cancer (GC) and peptic ulcer disease (PUD) are common diseases of the gastrointestinal tract happening worldwide $(1,2)$. Gastric cancer is the fourth and fifth most prevalent cancer in men and women, respectively, and the second cause of cancer- related death (3). A number of risk factors including diet, lifestyle, genetic and socioeconomic factors are introduced for gastric carcinogenesis and PUD development which infection with Helicobacter pylori is the mostly specified $(4,5)$. Helicobacter 
pylori can cause chronic inflammation of the gastric mucosa which is the initial step in gastric ulcer disease, gastric cancer and duodenal ulcer disease (6). Along with the pro-tumorigenic effects resulting from chronic inflammation, $H$. pylori influences the regulation of the host immune responses in favor of tumor cells (7). $H$. pylori binds to gastric epithelium mainly through its BabA virulence factor and then begins the colonization process in which urease enzyme plays an important role in this stage. Following colonization, other virulence factors such as $\mathrm{CagA}, \mathrm{VacA}$ and urease are involved which lead to disruption in epithelial barrier and mucus layer resulting cellular damage (8).

Galectins are a major group of lectins superfamily which play substantial roles in various biological functions by interacting with particular glycoconjugates (9). Galectin-9 (Gal-9) is a new member of the galectin proteins which was originally introduced as an eosinophil chemoattractant and activation factor (10) and is broadly expressed on different immune cells including T-cells, B-cells, macrophages, mast cells as well as epithelial and endothelial cells and fibroblasts (11). Gal-9 modulates tumor cell aggregation, adhesion, proliferation and apoptosis (12) and contributes to thymocyte apoptosis in mouse, suggesting a possible role in the process of negative selection occurring during $\mathrm{T}$ cell development (13). One of the main identified targets of Gal-9 is T-cell immunoglobulin and mucin domain-containing 3 (Tim-3) (14). The engagement of Tim-3 with Gal-9 leads to Th1 cell death and a consequent decline in IFN- $\gamma$ production leading to dys-regulation of the immune responses (15). Previous studies have demonstrated that high Gal-9 expression level contributes to cell aggregation during proliferation. MCF-7 subclones with higher Gal-9 expression formed induced tight clusters (16). Additionally, both cell aggregation and apoptosis of human melanoma cell lines were induced by exogenous Gal-9 which its protein was markedly expressed in melanocytic nevi while down-regulated in metastatic lesions (17). Despite many reports regarding Gal-9 up-regulation in cancer tissues, reduced Gal-9 expression has been noted in some other tumors (17-20). Analysis of the galectins expression in the human prostate cancer microenvironment delineated increased expression of Gal-1 and lower expression level of Gal-3, -4, -9, and -12 during disease promotion (18).

Although there are some reports about the expression profile of Gal-9 in gastric cancer from different geographical regions $(12,21)$, there is no data regarding its expression in Iranian patients with gastric cancer and especially in Mazandaran province where gastric cancer is a prevalent tumor. Additionally, there is no data about the Gal-9 expression pattern in patients with peptic ulcer disease. We have recently shown a high level of Tim$3 \mathrm{mRNA}$ and protein in biopsy sections of patients with GC and PUD) manuscript under review). Regarding the importance of $\mathrm{T}$ cell immunoregulatory mechanisms in gastric cancer and peptic ulcer disease and also various impacts of Gal-9 expression on tumor cells and cellular immune responses, this study aimed to evaluate the mRNA expression profile of Gal-9 in a wide range of gastric biopsies collected from patients with different gastric disorders to get more information about the immunoregulatory and pathogenesis mechanisms of these complications.

\section{Materials and Methods \\ Study Populations}

This case control study was performed using gastric biopsies obtained from 46 patients with $\mathrm{GC}, 44$ patients with PUD and 41 cases with non-ulcer dyspepsia (NUD) who underwent endoscopy for evaluation of their gastric problems at Imam Khomeini Hospital affiliated to Mazandaran University of Medical Sciences, Sari, Iran (Table 1).

Table 1. Major clinical and paraclinical findings of study populations

\begin{tabular}{lllll}
\hline & Variable & Non-Ulcer Dyspepsia & Peptic Ulcer Disease & Gastric Cancer \\
\hline Study samples (N) & Male & 11 & 18 & 36 \\
& Female & 30 & 26 & 10 \\
Age (year) & Mean \pm SD & $48.09 \pm 15.6$ & $56.69 \pm 15.6$ & $70.76 \pm 10.5$ \\
& Range & $19-77$ & $27-87$ & $50-90$ \\
\hline H. pylori infection $(\%)$ & Positive & 63.8 & 77.2 & 64.5 \\
& Negative & 36.2 & 22.7 & 35.4 \\
\hline
\end{tabular}


Disease diagnosis was done endoscopically and on the basis of morphologic and H\&E staining findings evaluated by an independent pathologist. According to the mentioned criteria, the patients were divided into three groups: patients with gastric cancer, patients with peptic ulcer and patients without any gastric ulcers named as non-ulcer dyspepsia control group. Gastric tissue biopsies were obtained from antrum and body of stomach in all three studied groups. Patients with any autoimmune diseases and those who received chemotherapy treatment were excluded from this study. The experimental procedure of the study was approved by the Ethics Committee and written informed consents were obtained from all the participants.

\section{Determination of H. pylori infection}

Rapid urease test was done for all samples when they referred to endoscopy examination. In addition, for GC and PUD patients, H\&E stained biopsy sections

\section{A}

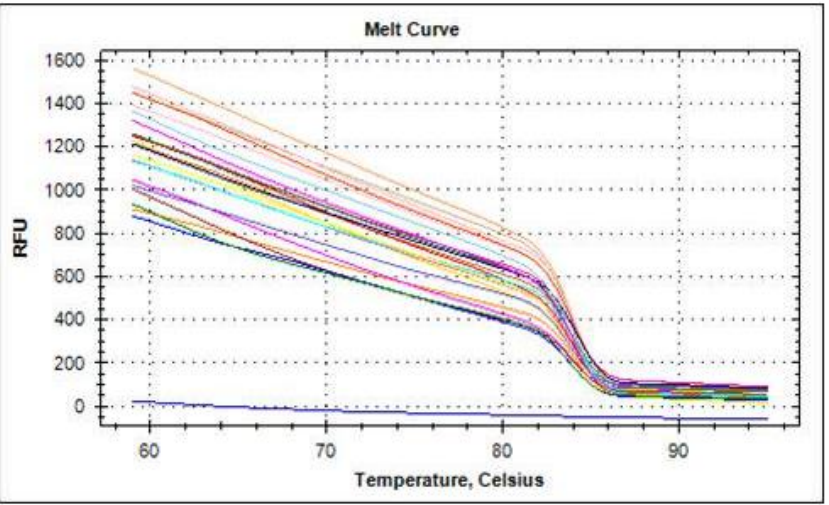

C

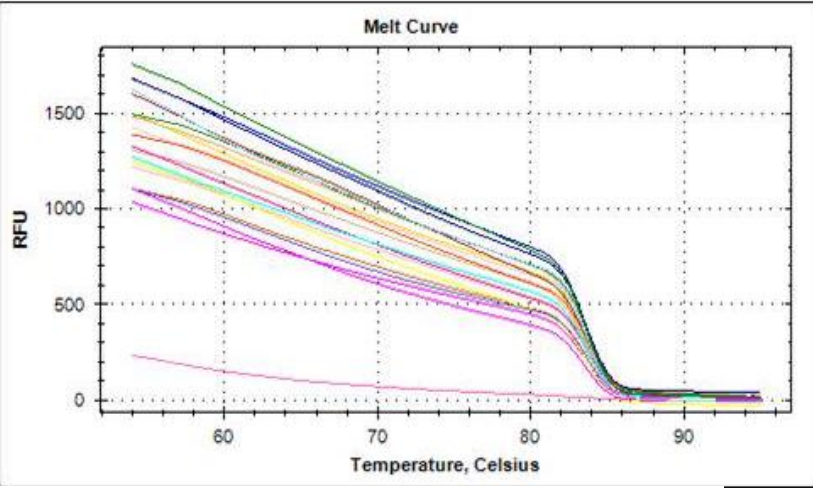

Figure 1. Melt curve and melt peak analysis of Gal-9 and $\beta$-actin

A and B. Melt curve and melt peak analysis of Gal-9 mRNA amplification by Real-time PCR, C and D. Melt curve and melt peak analysis of $\beta$ actin mRNA by Real-time PCR.

\section{Relative Quantitative Real-time PCR for detection of Gal-9}

Real-time PCR was performed using 2X Thermo Scientific Maxima SYBR Green/ROX qPCR Master were evaluated for $H$. pylori detection by a pathologist as well.

RNA extraction and cDNA synthesis

Total RNA was extracted from homogenized gastric tissue biopsies using a column-based Qiagen RNeasy Mini Kit (Qiagen, Germany) according to manufacturer's protocol. The quality of isolated nucleic acids was verified by nano-spectrophotometer (WPA, England) and electrophoresis in a 1.5\% agarose gel. Complementary DNA (cDNA) was reverse-transcribed from $1 \mu \mathrm{g}$ total RNA in a total volume of $20 \mu \mathrm{l}$ reaction mixture using the Thermo Scientific RevertAid first strand cDNA synthesis kit (Thermo Scientific, USA). The cDNA synthesis instruction was as follows: $1 \mu \mathrm{l}$ random hexamer, $4 \mu \mathrm{l}$ $5 \mathrm{x}$ reaction buffer, $1 \mu \mathrm{l}$ RNase inhibitor buffer, $2 \mu \mathrm{l}$ dNTP 10mM, $1 \mu$ l RevertAid M-MuLV Reverse Transcriptase and RNase/DNase free water at $25^{\circ} \mathrm{C}$ for $5 \mathrm{~min}, 42{ }^{\circ} \mathrm{C}$ for $1 \mathrm{~h}$ and $70{ }^{\circ} \mathrm{C}$ for $5 \mathrm{~min}$.

\section{B}

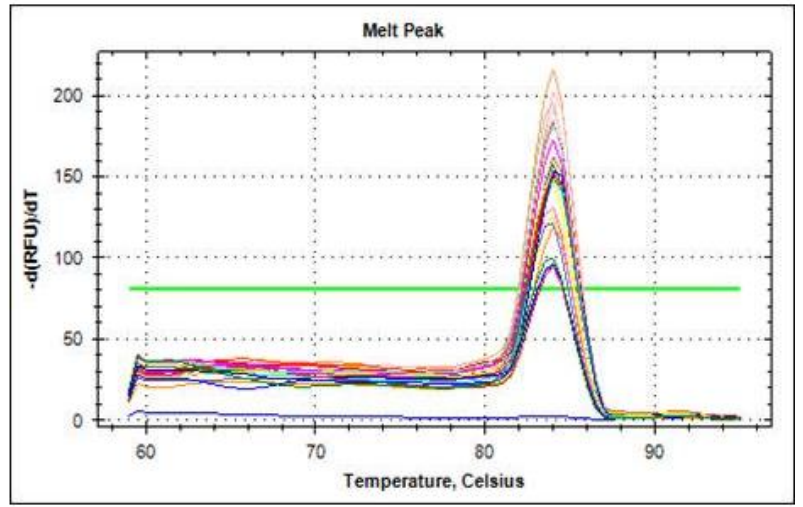

D

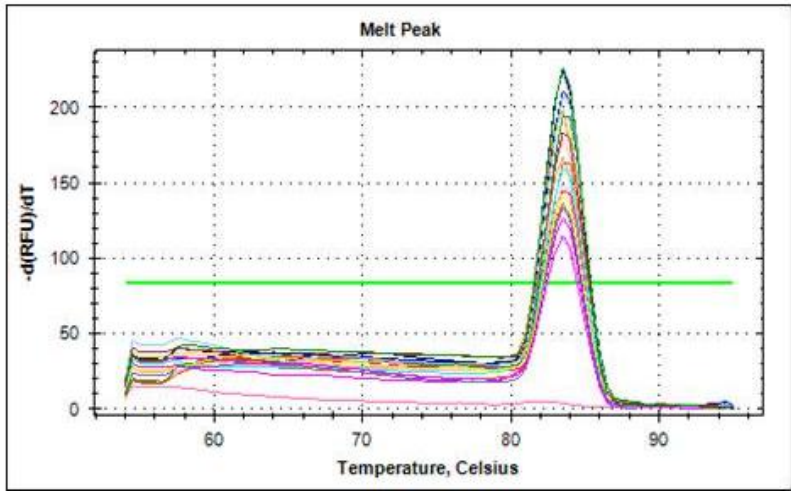

Mix (Thermo Scientific, USA) reagent in an iCycler iQ5 Real-time PCR system (Bio RAD, USA) with the following primers: Gal-9, forward: CAG TGC TCA GAG TTC CAC A, reverse: TGA GGC AGT GAG 
CTT CAC AC; $\beta$-actin, forward: CCT TCC TGG GCA TGG AGT CCT, reverse: TGG GTG CCA GGG CAG TGA T. The PCR reactions were amplified at $95{ }^{\circ} \mathrm{C}$ for initial denaturation followed by 40 cycles at $94{ }^{\circ} \mathrm{C}$ for 30 seconds, $59^{\circ} \mathrm{C}$ (Gal-9) and $57{ }^{\circ} \mathrm{C}$ ( $\beta$-actin) for 30 seconds, and $72{ }^{\circ} \mathrm{C}$ for 30 seconds. The PCR amplicon sizes were $118 \mathrm{bp}$ and 174 bp for gal-9 and $\beta$-actin, respectively. Each run was completed with a melting curve analysis to confirm the specificity of the amplification and the absence of primer dimers. Relative expression level of Gal-9 mRNA was determined with $2^{-\Delta C t}$ value using $\beta$-actin as an internal housekeeping gene.

\section{Statistical analysis}

SPSS 21 for Windows was used for all statistical analyses. One-Way ANOVA was applied to compare the statistical difference between the three groups and Mann-Whitney $U$ test or Independent sample t-test was applied to compare differences between two groups. The Spearman Rank and Pearson correlation tests were appropriately applied to analyze the correlation of Gal-9 expression with the clinical parameters of the patients. In addition, statistical power was also calculated. $\mathrm{p}<0.05$ was considered significant.

\section{Results}

Expression of Gal-9 mRNA in gastric tissues obtained from patients with gastric cancer, peptic ulcer and non-ulcer dyspepsia

Gal-9 mRNA expression in gastric tissues from three studied groups was evaluated using relative quantitative Real-time PCR assay. To calculate the relative expression of Gal-9, the $\beta$-actin gene was also amplified in all the samples and was considered as the internal control. To more validate and check the reproducibility of the data obtained from our optimized Real-time PCR method, intra-assay and inter-assay experiments were performed for both Gal9 and $\beta$-actin genes. Coefficient of variation (CV) indices of intra-assay and inter-assay analysis were 0.007 and 0.005 for Gal-9 and 0.03 and 0.02 for $\beta$ actin, respectively. In addition, $\mathrm{Ct}$ values obtained from $\beta$-actin expression as the housekeeping gene in different biopsy samples showed low and acceptable variations. The primer efficiencies were defined as $100.4 \%$ and $90.4 \%$ for Gal-9 and $\beta$-actin, respectively. The melt curve and melt peak analysis confirmed the specific expression of both Gal-9 and $\beta$-actin (Figure 1). As shown in figures $2 \mathrm{~A}$ and $2 \mathrm{~B}$, Gal-9 mRNA was similarly expressed in gastric biopsies obtained from patients with GC and NUD (2- $\Delta \mathrm{Ct}=0.022$ vs $0.0144, \mathrm{p}=0.30$, SP: 0.15$)$. In addition, to explore Gal-9 expression in other pathological chronic inflammation state of the stomach, Gal-9 mRNA expression in peptic ulcer disease was also analyzed. However, no significant difference was observed between Gal-9 mRNA expression in gastric tissues obtained from patients with PUD compared to NUD stomach tissues $\left(2^{-\Delta \mathrm{Ct}}\right.$ $=0.088$ vs $0.144, \mathrm{p}=0.16$, SP: 0.15 , Figures $2 \mathrm{~A}$ and 2B). No significant correlations were found between Gal-9 mRNA expression and different clinicopathological features repsented in Table 1.
A

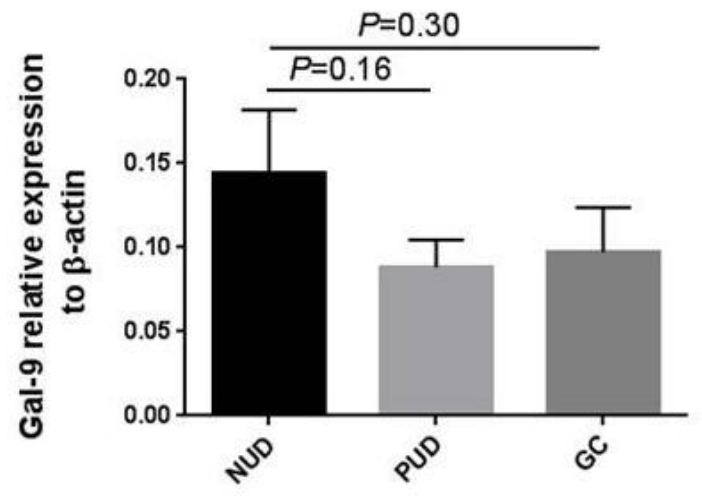

B

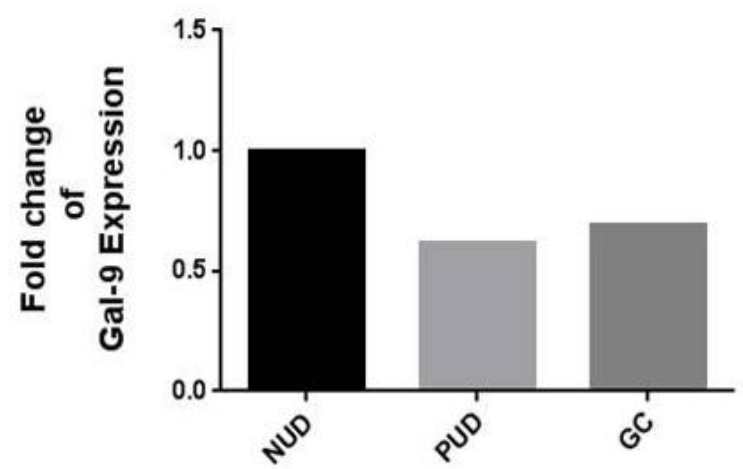

Figure 2. Similar expression of Gal-9 mRNA in gastric biopsies

A. Relative expression of Gal-9 mRNA in gastric biopsies obtained from cases with gastric cancer, peptic ulcer disease and non-ulcer dyspepsia. B. Fold increase of Gal-9 expression in patients with gastric cancer and peptic ulcer disease compared to non-ulcer dyspepsia group who served as control. Relative expression of Gal-9 is represented as $2-\Delta \mathrm{Ct}$ value after normalization with $\beta$-actin as an internal control. The $\mathrm{Y}$ axis shows the Mean+SEM value. 
Gal-9 is similarly expressed in H. pylori positive and negative samples

From 131 participants tested for H. pylori infection, the total positive rate of $H$. pylori infection was $69.3 \%$. Infection with $H$. pylori was confirmed in $64.5 \%, 77.2 \%$ and $63.8 \%$ of cases with GC, PUD and NUD, respectively. No significant correlation was found between Gal-9 expression and infection with H. pylori in any of the three studied groups (Figures $3 \mathrm{~A}$ and $3 \mathrm{~B}$ ). Moreover, when all the participants were divided into two $H$. pylori positive and negative groups, no significant difference for Gal-9 expression was found either $\left(2^{-\Delta \mathrm{Ct}}\right.$ of patients with and without $H$. pylor $i$ infection $=0.1$ vs $0.129, \mathrm{p}=0.51, \mathrm{SP}: 0.16$ ).

A

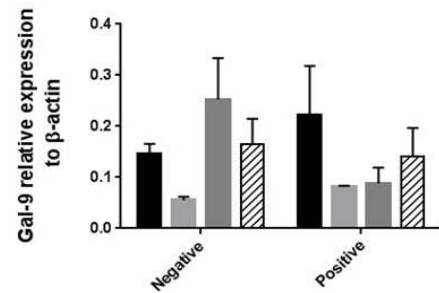

- Non-Ulcer Dyspepsia - Gastric Cancer [7] All Subjects

B

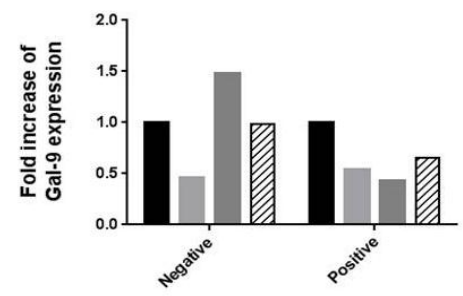

Non- Ulcer Dyspepsi Peptic Ulcer Disease Gastric Cancer EZ All Subjects

Figure 3. Correlation of Gal-9 mRNA expression with Helicobacter pylori infection

A. Similar Gal-9 mRNA expression in H. pylori positive and negative cases affected with gastric cancer, peptic ulcer or nonulcer dyspepsia. All enrolled samples were also divided in two groups based on the $\mathrm{H}$. pylori infection. No significant difference of Gal-9 expression was found between patients with or without $\mathrm{H}$. pylori infection. B. Fold increase of Gal-9 expression in H. pylori positive and negative cases. Relative expression of Gal-9 is represented as $2^{-\Delta \mathrm{Ct}}$ value after normalization with $\beta$-actin as an internal control. The $\mathrm{Y}$ axis shows the Mean+SEM value.

\section{Discussion}

Modulation of immune response mechanisms through immune checkpoint regulatory molecules has a great impact on the outcome and severity of different chronic inflammation diseases (22). To date, there are a lot of studies on the importance of Gal-9 expression and possible immune response skewness by Gal9/Tim-3 interaction as a main immune checkpoint pathway in the context of cancers and chronic infections (23). Since a better understanding of the Tim-3 in the mechanisms responsible for progressive T-cell dysfunction and exhaustion has been emerged, there has been considerable interest in the evaluation of Tim-3 and Gal-9 in different chronic inflammation conditions (24). Based on our previous study regarding up-regulation of Tim-3 mRNA and protein in gastric biopsies obtained from gastric cancer and peptic ulcer patients (submitted manuscript), in the current study, the expression pattern of Gal-9 as Tim3 major ligand was explored in patients with GC and PUD together with NUD as control group. Unlike Tim-3, Gal-9 was similarly expressed in gastric biopsies collected from three studied groups which suggests that Gal-9 is not involved in these chronic inflammation conditions.

Literature review shows that there are controversial reports on Gal-9 expression profile in different malignancies. While some studies rely on decreased Gal-9 expression in prostate cancer (18), melanoma (17), hepatocellular carcinoma (19), cervical intraepithelial neoplasia and cervical squamous cell carcinoma (20), there are some studies which have demonstrated up-regulation of Gal-9 in oral cancer (25), pancreatic cancer (26) and Hodgkin lymphoma (27). However, the expression of Gal-9 was significantly higher in patients with breast cancer and was suggested to be an antimetastatic potential factor by inhibiting invasion to extracellular matrix, detachment from tumors, and attachment to vascular endothelium (16). The only two other reports on Gal9 expression pattern in gastric cancer also appear to be conflicting. In a study by Jiang $\mathrm{J}$ et al. Gal-9 protein was more detected in gastric cancer than in normal mucosa. But, down-regulation of Gal-9 was found to be associated with tumor invasion and metastasis (21). In a later study, decreased expression of Gal-9 was reported in GC tissues when comparing with normal mucosa (12). Interestingly, our results are indicating similar expression of Gal-9 in patients with gastric cancer compared to control group. In addition, unchanged concentration of Gal-9 in sera of patients with breast cancer and healthy controls was previously reported (28). Considering that cancerous tissues are known to reveal an altered galectin expression profile and galectins are able to be secreted, so changes in the levels of circulating galectins are really informative (29). Like galectin-8, galectin-9 mRNA transcripts have extensive splicing too, and this complicates the interpretation of the studies in which no distinction between these splice variants has been made. Additionally, differences between patient populations could just as well reflect a switch in isoform expression and cause these controversial reports on Gal-9 expression in gastric cancer (29). Tim-3 over-expression in GC patients suggests the roles of other Tim-3 ligands such as high-mobility group box 1 protein (HMGB1) and phosphatidylserine and not Gal-9 in the context of 
GC immune regulation. Over-expression of HMGB1 and phosphatidylserine has been reported in some cancers and was associated with metastasis progression (30-32). In concordance with these interpretations, Chiba et al. demonstrated that the regulation of nucleic acid-mediated innate immune responses by Tim-3 is dependent on HMGB1 but not on Gal-9 or phosphatidylserine (33). Surface exposure of phosphatidylserine by malignant cells was shown in tumorigenic Friend erythroleukemia cells (34), some leukemia and neuroblastoma cells (35), malignant melanoma (36) and human gastric carcinoma cells (37) which serve as uniform marker of tumor cells and metastasis as well as a target for novel therapeutic approaches (32). In the case of gastric cancer, phosphatidylserine-positive platelets, leukocytes, and microparticles were significantly higher in patients with tumor-node-metastasis stage III/IV gastric cancer than those in stage I/II patients or healthy controls (38). Then, it seems that more comprehensive and additional studies are required to clarify the regulation of Gal-9 expression in malignant transformation.

Regarding the common etiologic causes of GC and PUD and the fact that PUD is a pathological chronic inflammation of the stomach, the Gal-9 mRNA expression was also evaluated for the first time in PUD patients to suggest that Gal-9 expression may contribute to modulation of the local immune responses in PUD development. No significant difference of Gal-9 mRNA expression between patients with PUD and control group suggests that Gal-9 may not be involved in immunopathogenesis of peptic ulcer disease, although further studies are needed to more confirm this finding.

Besides all environmental factors, infection with $\mathrm{H}$. pylori is the most important predisposing factor for development of gastric cancer and peptic ulcer disease. However, we did not find any significant correlation between Gal-9 mRNA expression and $\mathrm{H}$. pylori infection. The same finding has also been reported by a recent study indicating the similar Gal9 expression in $H$. pylori positive and negative patients with gastric cancer (21). Different variants of H. pylori have various effects on host immune responses and engage different immune regulatory molecules (39). The presence or absence of some crucial virulence factors such as cagA is associated with the development of severe gastritis, atrophic gastritis, GC and PUD (40). Therefore, it seems that different properties of $H$. pylori strains in the activation of immune system mechanisms may influence the recruitment of the various immune cells expressing immune regulatory molecules like Gal-9. Besides our experimental findings, considering some complementary experiments would help to clarify the
Gal-9 expression pattern and its precise role in pathogenesis of GC and PUD. For instance, expression evaluation of different variants of Gal-9 and measurement of Gal-9 protein expression are suggested for further investigation.

In conclusion, similar expression of Gal-9 in gastric tissues from patients with GC and PUD in comparison with NUD group suggests no possible role of this molecule on tumorigenesis and immunoregulatory mechanisms of these gastric pathological problems. Consideration of other Tim-3 ligands is suggested for further studies to more understand the immune regulation mechanisms of GC and PUD.

\section{Acknowledgements}

This work was supported by a grant from Mazandaran University of Medical Sciences (No: 1412). The authors thank Dr. Abolghasem Ajami and Dr. Mohsen Tehrani for their invaluable consultation.

\section{Authors' contributions}

NM performed the experiments and wrote the manuscript; TT selected the patients and consulted as gastroenterologist; AH designed the project, involved in optimization of all experiments and made the final revision of the manuscript.

\section{Conflict of interest}

The authors state no conflict of interest.

\section{References}

1. Lao S, Caldas C, Fitzgerald R. Genetic predisposition to gastrooesophageal cancer. Curr Opin Genet Dev. 2010; 20(3): 210-217 PMID: 20347291

2. Siegel RL, Miller KD, Jemal A. Cancer Statistics, 2061. CA Cancer J Clin 2016; 66(1):7-30. PMID: 26742998

3. Mousavi SM, Gouya MM, Ramazani R, et al. Cancer incidence and mortality in Iran. Ann Oncol. 2009; 20(3):556-63. PMID: 19073863

4. Malekzadeh R, Derakhshan MH, Malekzadeh Z. Gastric cancer in Iran: epidemiology and risk factors. Arch Iran Med. 2009; 12(6):576-83. PMID: 19877751

5. Correa P. Human gastric carcinogenesis: A multistep and multifactorial process-first American Cancer Society award lecture on cancer epidemiology and prevention. Cancer Res. 1992; 52(24):6735-40. PMID: 1458460

6. Shikata K, Doi Y, Yonemoto K, et al. Population-based prospective study of the combined influence of cigarette smoking and Helicobacter pylori infection on gastric cancer incidence the Hisayama study. American Journal of Epidemiology 2008; 168(12):1409-15. PMID:18945691

7. Shokrzadeh M, Karami M, Ghadi MAE. Measuring organophosphorus insecticide residue in rice produced in Amol, north of Iran. J Mazandaran Univ Med Sci. 2013; 23-1, 215-21p 
8) Slattery ML, Edwards SL, Samowitz W, Potter J. Associations between family history of cancer and genes coding for metabolizing enzymes (United States). Cancer Causes Control 2000; 11(9):799-803.

9. Kim JW, Lee CG, Park YG, et al. Combined analysis of germline polymorphisms of p53, GSTM1, GSTT1, CYP1A1, and CYP2E1. Cancer 2000; 88(9):2082-91. PMID: 10813720

10 Tan XL, Spivack SD. Dietary chemoprevention strategies for induction of phase II xenobiotic-metabolizing enzymes in lung carcinogenesis: a review. Lung Cancer 2009; 65(2):129-37. PMID: 19185948

11. Nebert DW, Dalton TP. The role of cytochrome P450 enzymes in endogenous signaling pathways and environmental carcinogenesis. Nat Rev Cancer 2006; 6(12):947-60. PMID: 17128211

12. Boccia S, Sayed-Tabatabaei FA, Persiani R, et al Polymorphisms in metabolic genes, their combination and interaction with tobacco smoke and alcohol consumption and risk of gastric cancer: a case-control study in an Italian population. Bio Medicine Cancer 2007; 7:206

13. Gonzalez FJ, Kimura S. Understanding the role of xenobiotic metabolism in chemical carcinogenesis using gene knockout mice. Mutat Res 2001; 477(1-2):79-87. PMID: 11376689

14. Cai L, Yu SZ, Zhan Z. Cytochrome P450 2E1 genetic polymorphism and gastric cancer in Changle, Fujian Province. World J Gastroenterol. 2001; 7:792-5. PMID: 11854903

15. Guengerich FP. Roles of cytochrome P-450 enzymes in chemical carcinogenesis and cancer chemotherapy. Cancer Res 1988; 48(11):2946-54. PMID: 3284639

16. Nair PC, McKinnon RA, Miners JO. Cytochrome P450 structure-function: insights from molecular dynamics simulations. Drug Metab Rev 2016; 48(3): 3, 434-52. PMID: 27167388

17. Bartsch H, Montesano R. Relevance of nitrosamines to human cancer. Carcinogenesis 1984; 5(11):1381-93. PMID: 6386215

18. Guengerich FP, Kim DH, Iwasaki M. Role of human cytochrome P-450IIE1 in the oxidation of many low molecular weight cancer suspects. Chem Res Toxicol 1991; 4(2):168-79. PMID: 1664256

19. Lieber CS. Cytochrome P4502E1: its physiological and pathological role. Physiol Rev. 1997; 77(2):517-44. PMID: 9114822

20. Umeno M, McBride OW, Yang CS, Gelboin HV, Gonzalez FJ. Human ethanol-inducible P450IIE1: complete gene sequence, promoter characterization, chromosome mapping, and cDNAdirected expression. Biochemistry 1988; 27(25):9006-13. PMID: 3233219

21. Gao CM, Takezaki T, Wu JZ, et al. CYP2E1 RsaI polymorphism impacts on risk of colorectal cancer association with smoking and alcohol drinking. World J Gastroenterol. 2007; 13(43):5725-30. PMID: 17963298

22. Hayashi SI, Watanabe J, Kawajiri K. Genetic polymorphisms in the 5'-flanking region change transcriptional regulation of the human cytochrome P450IIE1 gene. J Biochem. 1991; 110(4):55965. PMID: 1778977

23. Morita M, Le Marchand L, Kono S, et al. Genetic polymorphisms of CYP2E1 and risk of colorectal cancer: The fukuoka colorectal cancer study. Cancer Epidemiol Biomarkers Prev. 2009; 18(1):235-4. PMID: 19124503

24. Jemal A, Bray F, Center MM, et al. Global cancer statistics. CA Cancer J Clin. 2011; 61(2):69-90. PMID: 21296855

25. Mohebbi M, Mahmoodi M, Wolfe R, et al. Geographical spread of gastrointestinal tract cancer incidence in the Caspian Sea region of Iran: spatial analysis of cancer registry data. BMC Cancer 2008; 8:137. PMID: 18479519

26. Glatt HR. Overview of bioactivation of chemical carcinogens. Biochem Soc Trans. 2000; 28(2):1-6. PMID: 10816089

27. Cai L, Zheng ZL, Zhang ZF. Cytochrome p450 2E1 polymorphisms and the risk of gastric cardia cancer. World $\mathrm{J}$ Gastroenterol 2005; 11(12): 1867-71. PMID: 15793883

28. Tang K, Li X, Xing Q, et al. Genetic polymorphism analysis of cytochrome P4502E1 (CYP2E1) in Chinese Han populations from four different geographic areas of Mainland China. Genomics 2010; 95(4):224-9. PMID: 20100563

29. Babaei M, Pourfarzi F, Yazdanbod A, et al. Gastric cancer in Ardabil, Iran-A review and update on cancer registry data. Asian Pac J Cancer Prev. 2010; 11: 595-9. PMID: 21039022

30. Ghorbani A, Hosseini V, Ajami A, et al. Association between polymorphism of interleukin 17 (IL-17F) and increased susceptibility to gastric cancer. J Mazandaran Univ Med Sci. 2012; 22: $11-20$

31. Biglarian A, Hajizadeh E, Govhari M, Khodabakhshi R Survival analysis of patients with gastric cancer and its related factors. Kosar Med J 2008; 12(4): 345-55.

32. Feng J, Pan X, Yu J, et al. Functional PstI/RsaI polymorphism in CYP2E1 is associated with the development, progression and poor outcome of gastric cancer. PloS One. 2012; 7(9):e44478. PMID: 22957075

33. Bolt HM, Roos PH, Thier R. The cytochrome P-450 isoenzyme CYP2E1 in the biological processing of industrial chemicals: Consequences for occupational and environmental medicine. Int Arch Occup Environ Health 2003; 76(3):174-85. PMID: 12690492

34. Gonzalez A, Ramirez V, Cuenca P, Sierra R. Polymorphisms in detoxification genes cyp1A1, cyp2E1, GSTT1 and GSTM1 in gastric cancer susceptibility. Rev Biol Trop. 2004; 52(3):591-600. PMID: 17361553

35. Kato S, Naito Z, Matsuda N, et al. Localization of cytochrome $\mathrm{P} 4502 \mathrm{E} 1$ enzyme in normal and cancerous gastric mucosa and association with its genetic polymorphism in unoperated and remnant stomach. J Nippon Med Sch. 2011; 78(4):224-34. PMID: 21869556

36. Tsukino H, Kuroda Y, Qiu D, et al. Effects of cytochrome P450 (CYP) 2A6 gene deletion and CYP2E1 genotypes on gastric adenocarcinoma. Int $\mathrm{J}$ Cancer. 2002; 100(4):425-8. PMID: 12115524

37. Shahriary GM, Galehdari H, Jalali A, et al. CYP2E $1 * 5 B$, CYP2E1*6, CYP2E1*7B, CYP2E1*2, and CYP2E1*3 allele frequencies in Iranian populations. Asian Pac J Cancer Prev. 2012; 13:6505-10. PMID: 23464483 